\title{
Effect of enzyme-extracted brewers' spent grain protein hydrolysates on inflammatory response in cells associated with atherosclerosis
}

\author{
D.J. Crowley ${ }^{1}$, Y.C. O’Callaghan ${ }^{1}$, A. Connolly ${ }^{2}$, R.J. FitzGerald ${ }^{2}$ and N.M. O’Brien ${ }^{1}$ \\ ${ }^{1}$ School of Food and Nutritional Sciences, University College Cork, Ireland and ${ }^{2}$ Department of Life Sciences, \\ University of Limerick, Ireland
}

Inflammation is an essential process in the body's reaction to nonlethal injury; however, excessive and uncontrolled inflammatory responses can lead to chronic diseases ${ }^{(1)}$. Several peptides derived from cereal sources including brewers' spent grain (BSG), a byproduct of the brewing industry, have demonstrated anti-inflammatory effects in vitro. Research from our laboratory has previously demonstrated that alkaline-extracted BSG protein hydrolysates possess anti-inflammatory activity in human Jurkat T cells ${ }^{(2)}$. The aim of this research was to investigate the ability of enzyme-extracted BSG protein hydrolysates to inhibit release of pro-inflammatory cytokines in stimulated macrophage and $\mathrm{T}$ lymphocyte cells.

Ten enzyme-extracted BSG protein hydrolysates (A-J) were investigated. The effect of the BSG protein hydrolysates on cell proliferation was assessed using the MTT assay and non-toxic concentrations of $0.005 \%(\mathrm{w} / \mathrm{v}$ ) and $0.001 \%$ (w/v) were selected for the investigation of cytokine production in conA-stimulated Jurkat T lymphocyte cells and LPS-stimulated RAW 264.7 macrophages, respectively. Cytokine production was measured by ELISA following a 24 hour incubation with BSG protein hydrolysates.

\begin{tabular}{|c|c|c|c|c|c|c|c|c|}
\hline \multicolumn{9}{|c|}{ Cytokine Production ( $\%$ of control) } \\
\hline & \multicolumn{4}{|c|}{ Jurkat T cells } & \multicolumn{4}{|c|}{ RAW $264 \cdot 7$ cells } \\
\hline & \multicolumn{2}{|c|}{ Interleukin-6 } & \multicolumn{2}{|c|}{ Interferon- $\gamma$} & \multicolumn{2}{|c|}{ Interleukin-6 } & \multicolumn{2}{|c|}{$\begin{array}{c}\text { Tumor Necrosis } \\
\text { Factor- } \alpha\end{array}$} \\
\hline & Mean & se & Mean & se & Mean & se & Mean & se \\
\hline Control & $100 \cdot 0$ & $0 \cdot 0$ & $100 \cdot 0$ & $0 \cdot 0$ & $100 \cdot 0$ & $0 \cdot 0$ & $100 \cdot 0$ & $0 \cdot 0$ \\
\hline A & $96 \cdot 6$ & $6 \cdot 3$ & $124 \cdot 3$ & $21 \cdot 5$ & $66 \cdot 1 *$ & $8 \cdot 9$ & $68 \cdot 4 *$ & $9 \cdot 9$ \\
\hline B & $101 \cdot 8$ & $5 \cdot 0$ & $104 \cdot 6$ & $8 \cdot 7$ & $82 \cdot 6$ & $5 \cdot 0$ & $91 \cdot 4$ & $7 \cdot 0$ \\
\hline $\mathrm{C}$ & $113 \cdot 9$ & $5 \cdot 1$ & $105 \cdot 7$ & $5 \cdot 0$ & $77 \cdot 5$ & $10 \cdot 5$ & $96 \cdot 9$ & $6 \cdot 6$ \\
\hline D & $100 \cdot 7$ & $6 \cdot 3$ & $99 \cdot 1$ & $9 \cdot 0$ & $76 \cdot 1$ & $4 \cdot 7$ & 92.7 & $7 \cdot 6$ \\
\hline E & $105 \cdot 4$ & 1.5 & 98.0 & $6 \cdot 5$ & $69 \cdot 7$ & $12 \cdot 0$ & 98.0 & $10 \cdot 1$ \\
\hline $\mathrm{F}$ & $99 \cdot 3$ & 3.4 & $73 \cdot 6$ & $12 \cdot 6$ & $85 \cdot 3$ & $10 \cdot 0$ & 91.8 & $7 \cdot 9$ \\
\hline G & $91 \cdot 5$ & $19 \cdot 8$ & $87 \cdot 6$ & $8 \cdot 0$ & $83 \cdot 5$ & 1.8 & 93.6 & $2 \cdot 8$ \\
\hline $\mathrm{H}$ & 89.9 & 11.5 & 81.7 & $16 \cdot 6$ & $58 \cdot 4^{*}$ & 11.8 & 73.6 & $10 \cdot 3$ \\
\hline I & $92 \cdot 1$ & $2 \cdot 2$ & $86 \cdot 7$ & $13 \cdot 8$ & 75.9 & 3.0 & 87.2 & 3.0 \\
\hline $\mathrm{J}$ & $66 \cdot 6^{*}$ & $10 \cdot 9$ & 77.9 & $16 \cdot 8$ & $61.4^{*}$ & $9 \cdot 3$ & 88.7 & $5 \cdot 1$ \\
\hline
\end{tabular}

Values are mean of three independent experiments. Statistical analysis by ANOVA followed by Dunnett's test. * Denotes significant difference $(\mathrm{P}<0 \cdot 05)$ in cytokine production relative to control.

BSG protein hydrolysates were more toxic in RAW 264.7 cells compared to Jurkat T cells, as determined by the MTT assay (data not shown). BSG protein hydrolysates A, H and J significantly $(\mathrm{P}<0 \cdot 05)$ decreased LPS-stimulated interleukin-6 (IL-6) production in RAW 264.7 cells. Hydrolysate A also significantly $(\mathrm{P}<0.05)$ decreased tumor necrosis factor- $\alpha(\mathrm{TNF}-\alpha)$ production in LPS-stimulated RAW 264.7 cells, while hydrolysate J significantly $(\mathrm{P}<0.05)$ inhibited IL-6 production in conA-stimulated Jurkat T cells. The results of this study suggest that selected enzyme-extracted BSG protein hydrolysates, particularly A and J, may be effective in reducing cytokine production in cells in culture and have potential to be developed as ingredients for functional foods aimed at combating chronic inflammation and related disorders such as atherosclerosis.

1. Chakrabarti S, Jahandideh F, Wu J. (2014) Biomed Res Int 2014, 608979.

2. McCarthy AL, O'Callaghan YC, Connolly A, et al. (2013) Food Funct 4, 1709. 